\title{
Influence of light-emitting diodes on phenylpropanoid biosynthetic gene expression and phenylpropanoid accumulation in Agastache rugosa
}

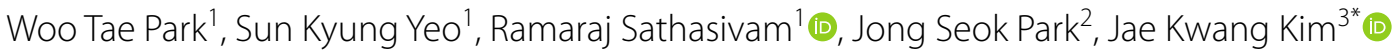
and Sang Un Park ${ }^{1 *}$

\begin{abstract}
Agsatache rugosa (Korean mint), belongs to the mint family and it has various medicinal properties. In addition, it has several valuable compounds such as monoterpenes and phenylpropanoid compounds. Amongst these, two compounds viz., rosmarinic acid (RA), and tilianin are well-known natural compounds that have numerous pharmacological properties. The phenylpropanoid biosynthetic gene expression under stress conditions and the subsequent accumulation of phenylpropanoid content has not been extensively studied in Korean mint. Here, we investigated the effect of light-emitting diodes (LEDs) on the expression levels of phenylpropanoid biosynthetic pathway genes and the accumulation of phenylpropanoid compounds such as RA and tilianin in A. rugosa. Real-time PCR analysis showed that the phenylpropanoid pathway genes responded to the LED lights. The transcript levels of downstream genes ( $C 4 H, C H S, C H I$, and RAS) were comparatively higher than those of upstream genes (PAL, TAT, and HPPR). In addition, HPLC analysis showed that the content of RA and tilianin were significantly higher in plants cultivated under white light than those grown under red, blue, green, and orange lights. The RA and tilianin content were the highest in the plantlets after three weeks of exposure to white light. These results suggested that white LED lights significantly enhanced the accumulation of phenylpropanoid compounds in A. rugosa.
\end{abstract}

Keywords: Agastache rugosa, LED lights, Phenylpropanoid biosynthetic genes, Tilianin, Rosmarinic acid

\section{Introduction}

Agsatache rugosa (Lamiaceae) is a perennial herb, which grows on slopes or roadsides in East Asian countries, including Korea, Japan, China, and Russia (Siberia). In Korea, the leaves of this ubiquitous herb have been generally used as a flavoring in certain traditional dishes, or as an herbal drug for traditional treatment of cholera,

\footnotetext{
*Correspondence: kjkpj@inu.ac.kr; supark@cnu.ac.kr

${ }^{1}$ Department of Crop Science, Chungnam National University, 99 Daehak-ro, Yuseong-gu, Daejeon 34134, South Korea

${ }^{3}$ Division of Life Sciences and Bio-Resource and Environmental Center,

Incheon National University, Incheon 22012, South Korea

Full list of author information is available at the end of the article
}

vomiting, inflammation, and oxidative stress-induced disorders [1-3]. This herb has also been reported to have antifungal, antiatherogenic, antitumor, HIV integrase inhibitory, and cytotoxic properties [4, 5]. A. rugosa contains a diverse variety of secondary metabolites, including sesquiterpenes, flavonoids, triterpenes, diterpenes, carotenoids, and some essential oils [6-8]. Among the different secondary metabolites, tilianin (a flavone glycoside) and rosmarinic acid (RA) are considered to have major medical properties $[4,9]$. Tilianin is gaining popularity among chemists and clinicians for its remarkable biological properties. It is reported that tilianin exhibits antihypertensive, anti-inflammatory, vaso-relaxant, and antiatherogenic effects $[10,11]$. RA is an important 
hydroxyl cinnamic acid ester and it is found in many medicinal plants [12]. The biosynthetic pathway of RA in plants has been investigated extensively because of its inevitable pharmacological, i.e., antibacterial, antiviral, antioxidant, and anti-inflammatory properties [13-15]. In the plant kingdom, RA protects plants against unfavorable conditions [12].

The proposed tilianin and RA biosynthetic pathways in A. rugosa are shown in Fig. 1. L-phenylalanine is the precursor of tilianin and RA. In the general phenylpropanoid pathway, phenylalanine is transformed into 4-coumaroyl-CoA through the activity of three enzymes such as phenylalanine ammonia-lyase $(P A L)$, cinnamate 4-hydroxylase $(C 4 H)$, and 4-coumarate:CoA ligase (4CL) [16]. Secondary metabolites like flavonoids, stilbenes, coumarin, and lignin are synthesized using the precursor molecule 4-coumaroyl-CoA. Chalcone synthase $(C H S)$ catalyzes the condensation of 4-coumaroyl-CoA with three malonyl-CoA molecules to generate naringenin chalcone [17]. Naringenin chalcone is converted into naringenin through a stereospecific isomerization reaction by accelerating flavonoid biosynthetic chalcone isomerase $(\mathrm{CHI})$ [18]. In the next step, through the activity of flavone synthase, apigenin is synthesized from naringenin [19]. Then, apigenin 4'-O-methyltransferase moves a methyl group to apigenin to produce acacetin [20]. Finally, glucosyltransferase change glucose to acacetin to synthesize tilianin

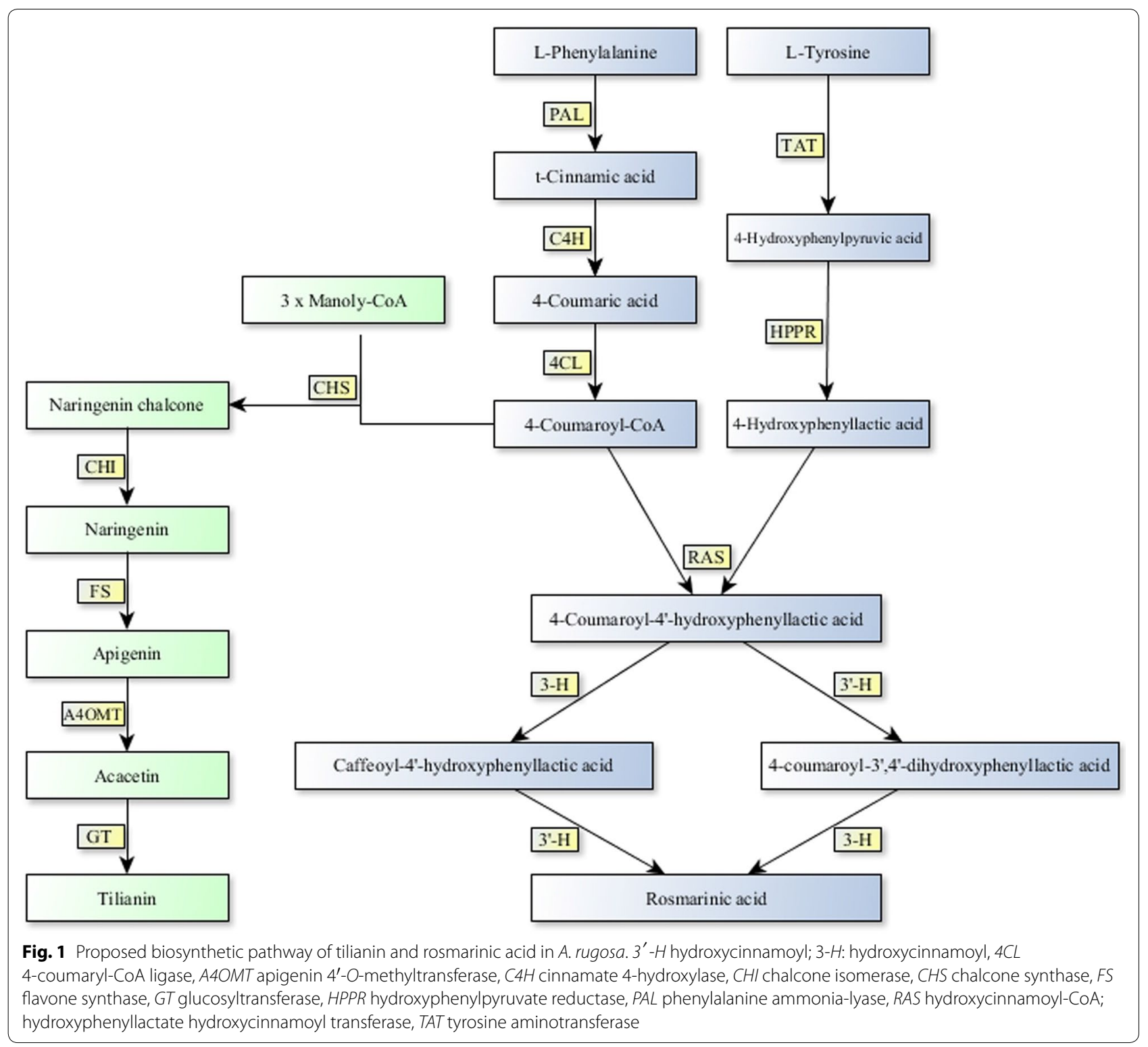


[21]. Condensation of 4-hydroxyphenyllactic acid with 4-coumaroyl-CoA is catalyzed by hydroxycinnamoylCoA:hydroxyphenyllactate hydroxycinnamoyltransferase $(R A S)$ to form 4-coumaroyl-4'-hydroxyphenyllactic acid, which is further converted to RA by two successive hydroxylation steps [22]. Two distinct cytochrome P450s, namely 3-hydroxycinnamoyl, and 3'-hydroxycinnamoyl are used as a catalyst for these metabolic reactions [23].

The quality and quantity of compounds found in medicinal plants vary due to several environmental factors like temperature, duration of sunlight, rainfall, soil, and exposure to light [24-26]. Light plays an important role in the growth and development of plants, as well as the regulation of gene expression and accumulation of metabolites. Plants have various photoreceptors to perceive different light waves. Phytochromes, which are red and far-red light photoreceptors, initiate seed germination, stimulates leaf expansion, and control flowering with other signaling pathways. Blue light photoreceptors, such as phototropins and cryptochromes, are involved in phototropism and opening of stomata with auxin signaling. Phototropism is the growth of plants in response to a light stimulus that helps plants absorb sunlight as much as possible. Several studies suggest that light-emitting diodes (LEDs) enhance the secondary metabolite accumulation in plants $[25,26]$. Although it is unclear why the specific LED wavelength affects specific genes, we presume that specific light wavelength is assimilated in different signaling pathways to harmonize stress adaptation response in plant growth and development.

The use of LEDs for facilitating plant growth in a controlled environment has many advantages, such as variation of light intensity or quality, high energy-exchange efficiency, longer lifetime, and low thermal energy output [27-30]. To date, no studies report how various LED wavelengths may affect the phenylpropanoid biosynthesis and the relative gene transcriptional level. Here, we investigated the influences of five different color LEDs (red, orange, green, blue, and white) on the phenylpropanoid accumulation and its transcriptional variation of the biosynthetic pathway genes, together with the variation in accumulation of RA and tilianin content in A. rugosa plantlets.

\section{Materials and methods}

\section{Plant material and LED treatments}

The seeds of $A$. rugosa were obtained from Aram seed company (Seoul, Korea). Before germination the seeds were surface-sterilized for $1 \mathrm{~min}$ with $70 \%(\mathrm{v} / \mathrm{v})$ ethanol, followed by $4 \%(\mathrm{v} / \mathrm{v})$ sodium hypochlorite solution by adding several drops of tween 20 for $10 \mathrm{~min}$ and then rinsed thrice using sterilized distilled water. The seeds were germinated on a plastic pot containing horticultural soil. The pots were placed in the growth chamber under a white fluorescent light/dark $(16 / 8 \mathrm{~h})$ photoperiod at $25{ }^{\circ} \mathrm{C}$. The germinated seeds were transferred into a room with controlled environmental conditions and it was maintained at $24{ }^{\circ} \mathrm{C}$ and $18 \pm 1{ }^{\circ} \mathrm{C}$ in day and night time, respectively, with approximately $50-70 \%$ relative humidity under five different LED light conditions. The seedlings of $A$. rugosa were subjected to five different monochromatic LEDs treatments; i.e., red $(645 \mathrm{~nm})$, orange $(590 \mathrm{~nm})$, green $(525 \mathrm{~nm})$, blue $(467 \mathrm{~nm})$, and white $(380 \mathrm{~nm})$ with $100 \pm 5 \mu \mathrm{mol} \mathrm{m}{ }^{-2} \mathrm{~s}^{-1}$ of photosynthetic photon flux density (PPFD) at the canopy level. The emission spectra of LED light sources are given in Additional file 1: Fig. S1. All the LED lights were purchased from Sammi electronic Co. Ltd, Seoul, Korea. The plantlets were harvested after 1,2 , and 3 weeks of LED exposure. All the harvested samples were immediately snap-frozen by using liquid nitrogen and then stored at $-80{ }^{\circ} \mathrm{C}$ until RNA isolation and/or high-performance liquid chromatography (HPLC) analysis.

\section{Total RNA extraction, cDNA synthesis, and quantitative real-time PCR}

The samples stored at $-80{ }^{\circ} \mathrm{C}$ were ground finely with liquid nitrogen by using a mortar and pestle. The extraction of total RNA was done using TRI reagent ${ }^{\circledR}$ (Molecular Research Center, Inc.). RNA integrity was measured using the NanoVue ${ }^{\mathrm{TM}}$ plus spectrophotometer (GE Healthcare, UK) and identified by using a formaldehyde RNA agarose gel. For first-strand cDNA synthesis, $1 \mu \mathrm{g}$ of total RNA was used for reverse transcription (ReverTra Ace- $\alpha-{ }^{\circledR}$ kit (Toyobo Co., Ltd, Osaka, Japan)). The resulting products were diluted 20 -fold and then used as a template for quantitative real-time PCR (qRT-PCR) with gene-specific primer (Table 1). qRT-PCR was performed by using a 2X Real-time PCR smart mix (Solgent, Korea) in a CFX96 real-time system (BIO-RAD Laboratories, USA). The thermal cycling conditions were set to $95^{\circ} \mathrm{C}$ for $15 \mathrm{~min}$ followed by $95^{\circ} \mathrm{C}$ for $15 \mathrm{~s}, 55^{\circ} \mathrm{C}$ for $15 \mathrm{~s}$ and finally at $72{ }^{\circ} \mathrm{C}$ for $20 \mathrm{~s}$ for 40 cycles. To quantify the expression level of genes involved in the phenylpropanoid pathway, the housekeeping gene, actin was used as an internal control.

\section{Analysis of phenylpropanoid compounds by HPLC}

For HPLC analysis samples were freeze-dried under vacuum for at least $48 \mathrm{~h}$. Then the samples were ground into a fine powder using mortar and pestle and the extraction was performed using the protocol described by Thwe et al. [31]. For phenylpropanoid analysis, $100 \mathrm{mg}$ of each sample was extracted by adding $5 \mathrm{~mL}$ of $100 \%$ methanol and incubating for $1 \mathrm{~h}$ at $60{ }^{\circ} \mathrm{C}$ in a sonicator. After centrifugation, the supernatant was filtered through a 
Table 1 Primers used to qRT-PCR analysis

\begin{tabular}{llll}
\hline Primers & Sequences $\left(\mathbf{5}^{\prime}\right.$ to $\mathbf{3}^{\prime}$ ) & $\begin{array}{l}\text { Accession } \\
\text { no. }\end{array}$ & Size (bp) \\
\hline ArActin $F$ & GGCCGTTCTCTCACTTTATGCTA & JX087435 & 122 \\
ArActin $R$ & ACCTCGAAATAGCATGGGGAAGT & & \\
ArPALF & ACGGCTCCAACGGTCATAATAAT & AF326116 & 108 \\
ArPAL $R$ & ATCCGCTTTACCTCCTCAAGGT & & \\
ArC4HF & GTTCGAGAGTGAGAATGATCCGT & AY616436 & 157 \\
ArC4HR & ATAATCCTTGAACAATTGCAGCC & & \\
ArCHSF & GACCAAAGCACCTATCCCGATTA & JQ314450 & 151 \\
ArCHSR & TTGGGTTCTCCTTCAGGTACTCC & & \\
ArCHIF & GCCTTCTCCAAAGATGGTTCTGT & JQ314451 & 194 \\
ArCHIR & TCTTGATTCAGTTTGCCTCAGC & & \\
ArHPPRF & AAGGGGATTAGGGTTACCAACACA & MT268327 & 200 \\
ArHPPRR & ATTCTACCCAATCCAATGATGCC & & \\
ArTATF & AGGCTGCAGTTCCTGAAATCATT & MT268329 & 163 \\
ArTATR & TTCACCATGAAAGCCATTGCTCC & & \\
ArRASF & GGCGAACTATCATACCCTGAG & MT268328 & 161 \\
ArRASR & AATCAATTTCCAGGCGTTTGCCG & & \\
\hline
\end{tabular}

$0.45 \mu \mathrm{m}$ PVDF filter (Whatman, GE Healthcare, UK) and subjected to HPLC (NS-4000, Futecs, Korea). HPLC analysis was carried out at $30^{\circ} \mathrm{C}$ by using an RP-HPLC column $(\mathrm{C} 18,250 \mathrm{~mm} \times 4.6 \mathrm{~mm}, 5 \mu \mathrm{m}$, Prontosil, Bischoff, Germany). The mobile phase was a gradient mixture of absolute methanol (solvent $\mathrm{A}$ ) and water containing $0.1 \%$ $(\mathrm{v} / \mathrm{v})$ acetic acid (solvent $\mathrm{B})$. The flow rate of the samples was $1 \mathrm{~mL} / \mathrm{min}$ under the following conditions: $15 \mathrm{~min}$, $45 \% \mathrm{~A} / 55 \% \mathrm{~B} ; 5 \mathrm{~min}, 55 \% \mathrm{~A} / 45 \% \mathrm{~B} ; 30 \mathrm{~min}, 80 \% \mathrm{~A} / 20 \%$ B; $10 \mathrm{~min}, 45 \% \mathrm{~A} / 55 \% \mathrm{~B}$. The chromatographic detection of all phenylpropanoids was performed at $340 \mathrm{~nm}$. A corresponding standard curve was used to quantify the phenylpropanoid content in the samples. Rosmarinic acid authentic standard was purchased from Sigma (Catalogue No.: BP1224, Sigma-Aldrich, Inc. St. Louis, USA), and the tilianin standard was obtained from the Korea Research Institute of Bioscience and Biotechnology (KRIBB), Daejeon, South Korea.

\section{Statistical analysis}

All the quantitative data were statistically analyzed by using Student's $t$ test at a significant level of 0.05 followed by IBM SPSS Statistics version 22.0 software (SSPS, Inc., Chicago, IL, USA) statistical software. All the experiments were executed in triplicates and the significant differences between treatments were assessed by the standard deviation.

\section{Results}

\section{The effect of LEDs on fresh weight content}

Three-week-old plantlets of $A$. rugose were exposed to five different colors of LED lights (i.e., red, orange, green, blue, and white). The fresh weight of plantlets gradually increased with increasing exposure time (Additional file 1: Fig. S2). Among the exposure times, the highest fresh weight was achieved in the 3rd week in all LED exposed plantlets when compared to the first 2 weeks. In the 3rd week of exposure, the highest fresh weight content was obtained in the plantlets exposed to red light followed by blue, orange, green, and white lights (Additional file 1: Fig. S2).

The effect of LEDs on phenylpropanoid biosynthetic genes expression

To determine the genomic effects of LEDs, using qRTPCR analysis, we analyzed the transcript levels of phenylpropanoid pathway genes in A. rugosa at 1,2, and 3 weeks of exposure to red, orange, green, blue, and white LEDs. The results showed that the LEDs greatly influenced the phenylpropanoid pathway genes, however, their expression pattern differed with the color of light. The expression pattern of the downstream pathway genes $(C 4 H$, $C H S, C H I$, and $R A S$ ) was higher than those of upstream pathway genes (PAL, TAT, and HPPR). Most of the phenylpropanoid pathway genes had a significantly higher expression in the plantlets exposed to white LEDs than in plantlets exposed to other LED irradiation.

The expression of $P A L$ was higher after 1 week of exposure when compared to two and 3 weeks. Among the LED treatments, the highest $P A L$ transcript was found in orange illumination, the expression level was $2.86,2.73$, 2.11-, and 1.40-fold higher than the red, blue, green, and white LEDs, respectively (Fig. 2). The $P A L$ gene expression showed a much higher variation in the second week of exposure. At this time, the $P A L$ gene expression was higher in the plantlets exposed to green LED, the expression level was 11.97, 9.67, 6.52, and 2.57-fold higher than the blue, orange, white, and red LEDs, respectively. Except for white LED exposure, the expression levels of $\mathrm{C} 4 \mathrm{H}$ increased sharply with increasing exposure time to the different colors of LED lights (Fig. 2). Among the treatments, white light resulted in the highest level of expression at one and two weeks of exposure, whereas after 3 weeks, the $\mathrm{C} 4 \mathrm{H}$ expression slightly decreased. At 3 weeks, orange irradiation showed a 2.11 -fold higher expression of $\mathrm{C} 4 \mathrm{H}$ than that of blue light exposure. The CHS transcript levels were considerably increased with increasing exposure time, except for the blue and orange illumination. Among the overall exposure, white and blue light showed the highest $C H S$ expression at 1 and 3 weeks of exposure, whereas, blue light showed 

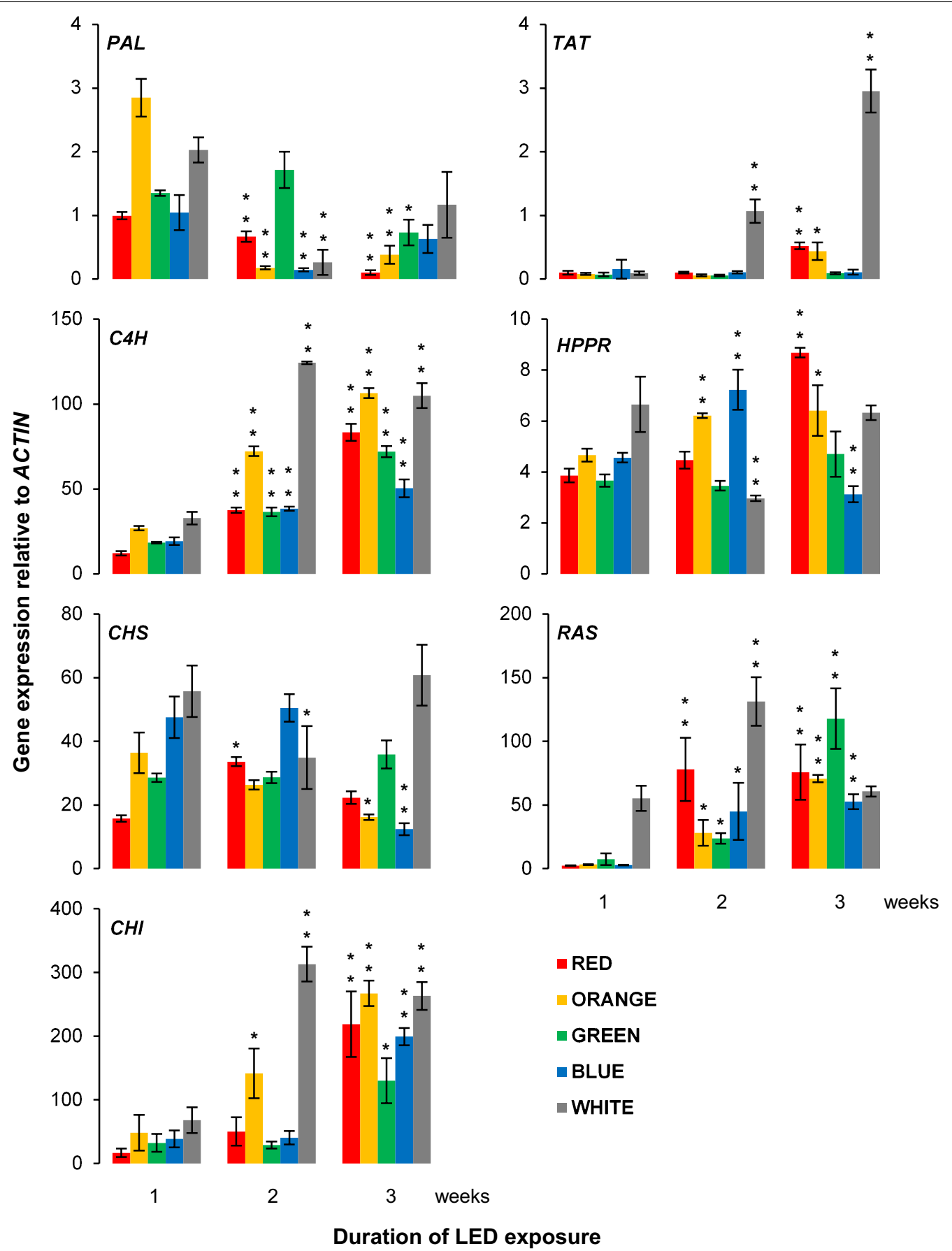

Fig. 2 Relative gene expression levels of phenylpropanoid biosynthetic genes in A. rugosa irradiated with different LED lights (red, orange, green, blue, and white). Relative fold changes were plotted after normalization to ACTIN7. Mean values and standard deviation were obtained from three independent biological experiments. Significant difference are highlighted with ${ }^{*} P<0.05$ and ${ }^{* *} P<0.01$

the decreased expression in the third week. However, all the LED exposure the $C H I$ gene was not significantly expressed at 1 week of exposure. In the 2 and 3 weeks of exposure, the expression level of $\mathrm{CHI}$ gradually increased with all LEDs (Fig. 2). Similarly, the transcript levels of
TAT were significantly expressed only in white light when compared with exposure to other LEDs. After 2 weeks of white light exposure, the TAT gene expression was 19.24, 18.87, 10.47, and 9.88 times higher when compared to green, orange, red, and blue LEDs, respectively. 
Furthermore, the expression level of HPPR showed different expressions between the light treatments, with the highest transcript level found at 1,2 and 3 weeks of exposure to white, blue and red LEDs, respectively. In the first week, white illumination resulted in the highest level of expression when compared to other treatments, whereas in the second week blue light showed 2.44 and 2.09 times higher expression of this gene when compared to white and green light, respectively. In the third week, plantlets exposed to red light showed 2.78 times higher expression than in blue light. The transcript levels of $R A S$ also gradually rose with increasing exposure times in different light colors. White LEDs increased RAS expression until the second week of exposure, and then gradually decreased (Fig. 2). The expression of RAS in the first week under white light was significantly higher than for the other illuminations, the expression level was $23.99,19.83$, 17.63, and 7.51 times higher than for red, blue, orange, or green lights, respectively. Similarly, in the second week, the expression level of this gene was higher under white light than other light treatments.

\section{The effect of LEDs on rosmarinic acid and tilianin in $A$. rugosa}

HPLC analysis of $A$. rugosa plantlets after treatment with different LED illumination revealed the presence of two phenylpropanoid compounds: RA and tilianin. The production of RA was influenced greatly by light treatments, with the highest observed accumulation at 3 weeks under white lights (Fig. 3). RA content in this treatment was $2.85,2.72,1.72$, and 1.71 times higher than in red, blue, green, or orange lights, respectively. In the second week of white light exposure, the accumulation of RA was also the highest and the level was 1.84, 1.50, 1.44, and 1.33 times higher than that with green, blue, orange, and red LEDs, respectively. The RA content obtained in white light exposed plantlets at a different time of exposure was 13.96 (first week), 41.96 (second week), and 47.14 (third week) $\mathrm{mg} / \mathrm{g}$ dry weight. The level of RA decreased in the third week in red, orange, and blue light exposure.

The tilianin content gradually increased with time in plantlets under most LED exposures (Fig. 4). In most cases, the level of tilianin was the highest at 3 weeks in the following order of LED exposure: white, orange, green, blue, and red. Under white illumination, the tilianin content was $1.79,1.57,1.32$, and 1.27 times higher levels than that in red, blue, green, and orange illumination, respectively. Tilianin level markedly increased after exposure to white light for 1, 2, and 3 weeks, with an increase of 4.58 , 6.74 , and $8.31 \mathrm{mg} / \mathrm{g}$ dry weight, respectively.

\section{Discussion}

LEDs have many advantages compared to existing artificial illumination light sources like metal-halide, fluorescent, and high-pressure sodium lamps for the growth of the plants especially in a controlled environment $[27,29,30]$. In this study, we found that LED lights significantly influenced the expression of phenylpropanoid biosynthetic genes in A. rugosa. The transcript levels of $P A L$ decreased slightly with increasing time of exposure in all types of LEDs. In contrast, in all the LED treatments the transcript levels of $C 4 H, C H I, T A T$, and $R A S$ genes increased gradually with increasing exposure time. Several studies have indicated that LEDs influenced the expression of secondary metabolite biosynthetic genes in various plants. At 2 days after exposure, the expression

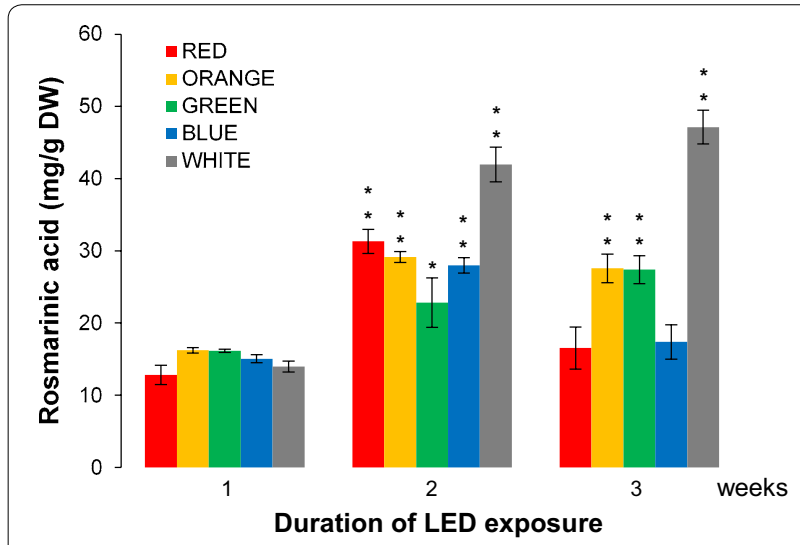

Fig. 3 Rosmarinic acid content in A. rugosa after exposes to various LED lights at different time intervals (1, 2, and 3 weeks). Each of the three individual experimental groups was analyzed by using HPLC. Error bars represent standard deviation values. Significant difference are highlighted with ${ }^{*} P<0.05$ and ${ }^{*} P<0.01$. DW, dry weight

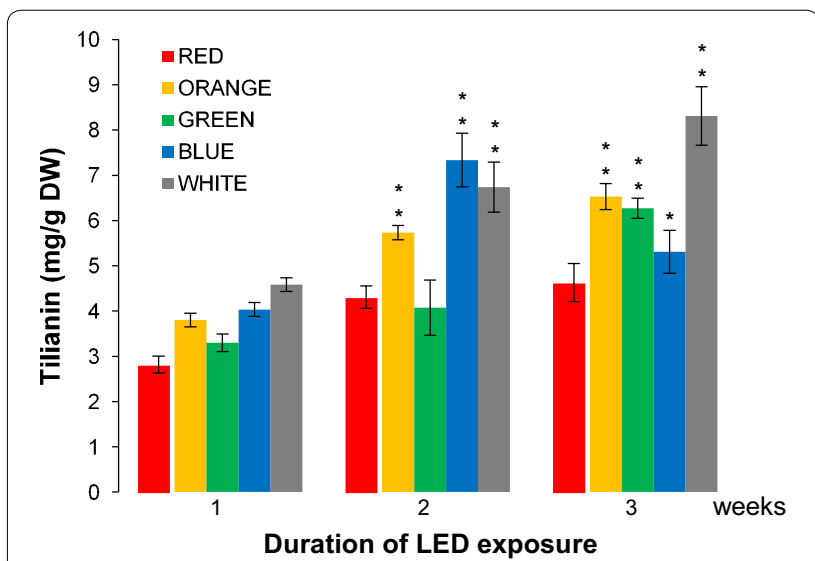

Fig. 4 Tilianin content in A. rugosa after expose to various LED lights at different time intervals (1,2, and 3 weeks). Each of the three individual experimental groups was analyzed using HPLC. Error bars show standard deviation values. Significant difference are highlighted with ${ }^{*} P<0.05$ and ${ }^{* *} P<0.01$. DW, dry weight 
of Ft4CL, FtCHS, FtCHI, FtFLS2, and FtDFR was comparatively higher in Fagopyrum tataricum sprouts grown under blue light than those cultivated under white or red LEDs [31]. A recent study reported that wheat sprouts exposure to red light resulted in a greater change in the expression level of TaPAL4, Ta4CL1, and Ta4CL5 genes [25]. Tuan et al. [32] reported that the level of carotenoid biosynthetic genes expression in $F$. tataricum sprouts was higher when grown in the presence of white light than blue and red LEDs. Similarly, in this study result, we found that the expression level of phenylpropanoid pathway genes was significantly varied among the different LEDs exposure. This indicates that different LEDs are capable of inducing a complex change in the expression of phenylpropanoid pathway genes in Korean mint.

Thwe et al. [31] found that catechin content in F. tataricum grown in red LED, significantly increased from 2 to 10 days after exposure; and the maximum catechin levels were detected at 10 days after exposure and the content was 9.6- and 43.1-fold higher when they are grown under blue and white LEDs, respectively. However, the highest rutin content ( $43.37 \mathrm{mg} / \mathrm{g}$ dry weight) was obtained after a 4-day exposure in sprouts under blue irradiation [31]. Similarly, sprouts grown under fluorescent light had a significantly higher amount of rutin than the content of other phenolic compounds [33]. This revealed that both LEDs and fluorescent lights can affect rutin content in sprouts. In contrast, in $F$. tataricum the production of chlorogenic acid did not vary significantly when grown under blue and white LEDs. Instead of chlorogenic acid, blue LEDs stimulated the production of anthocyanin [31]. Similarly, blue light enhanced the accumulation of secondary metabolic compounds in Norway spruce [34], lettuce [35], Chinese cabbage [36]. In tartary buckwheat, the phenolic compound cyanidin 3-O-glucoside was found only in sprouts raised in blue light, and its level did not vary during the sprouting process. After 10 days of exposure, the cyanidin 3-O-rutinoside level of F. tataricum grown under blue light was 2.8- and 10.6fold higher when compared to the sprouts grown under white and red LEDs, respectively [31]. Previously it was claimed that red light significantly enhanced the amount of carotenoids in citrus flavedo than other lights [37, 38]. A similar result was obtained in this study, that the different LEDs enhance the accumulation of rosmarinic acid and tilianin level in the Korean mint. All these reports suggest that LEDs of different colors have a positive effect on the accumulation of valuable secondary metabolites. These results might help to improve the content of specific medicinal compounds in Korean mint by growing under appropriate LED lights.

The expression of phenylpropanoid biosynthetic genes has been reported to be correlated with flavonoid content in plants [39-41]. The higher expression of the metabolic genes involved in phenylpropanoid biosynthetic pathways such as $A r C 4 H, A r P A L$, and $A r 4 C L$ might be responsible for increased accumulation of flavonoids, such as tilianin, and its precursor acacetin, in the leaves and flowers of $A$. rugosa [42]. Similarly, the lower expression of $\operatorname{ArPAL}, \operatorname{ArC4H}$, and $A r 4 C L$ might lead to trace accumulation of acacetin and tilianin in the roots. The expression levels of $\mathrm{ArCHS}$ and $\mathrm{ArCHI}$ were consistent with the accumulation of tilianin and acacetin in different organs of $A$. rugosa [42]. In this study, the gene (CHS and $C H I$ ) expression and tilianin levels were gradually increased with increasing exposure time. Therefore, this indicates that $\mathrm{CHS}$ and $\mathrm{CHI}$ may regulate the biosynthesis of acacetin and tilianin, as well as other flavonoids, in A. rugosa.

In medicinal plants, enzymes such as $\mathrm{PAL}, \mathrm{C} 4 \mathrm{H}$, and $4 \mathrm{CL}$ play a major role in the phenylpropanoid biosynthetic pathway are important for the production of RA. Especially, PAL is a key enzyme in the RA biosynthetic pathway in Salvia miltiorrhiza as the alteration of RA content directly correlates with $P A L$ expression [43]. Moreover, the downregulation of $P A L$ influences the expression of $C 4 H$ and $4 C L$ and leads to a reduction in rosmarinic acid content in S. miltiorrhiza [44]. The expression levels of $P A L$ and $4 C L$ correlate with their respective enzymatic activities and with the RA content in Melissa officinalis [45]. Three enzymes responsible for higher expression of the phenylpropanoid pathway genes in the leaves (ArPAL and $A r 4 C L)$ and flowers $(A r C 4 H)$ of $A$. rugosa, increase the accumulation of RA. The roots also showed a significant accumulation of RA in $A$. rugosa [42], although the expression of $A r P A L, A r C 4 H$, and $A r 4 C L$ was low. In contrast, high expression of $P A L$ in the roots of $S$. miltiorrhiza leads to a lower accumulation of RA [43]. In this study, the highest phenylpropanoid biosynthetic pathway genes expression leads to the relatively highest accumulation of rosamarinic acid in the Korean mint exposed to white LED. The reason for the increased expression of phenylpropanoid pathway genes and its accumulation in white LED light might be due to it can better penetrate the plant canopy when compared to other LED light for photosynthesis [46]. From this, we found that the biosynthesis of RA in plants is regulated by a complex mechanism.

Overall, this study demonstrates that the enzymes involved in the phenylpropanoid biosynthetic pathway, and two important pharmacological compounds of this pathway (RA and tilianin), can be modified by exposing A. rugosa plantlets to LEDs of different colors. Among these, white light was the most effective in inducing the synthesis of these phenylpropanoids in A. rugosa. This suggests that careful modulation of the light color might 
able to enhance the biomass production and compounds necessary for medicinal purposes, however, further studies are required for a wide range of assessments of these effects.

\section{Supplementary information}

Supplementary information accompanies this paper at https://doi. org/10.1186/s13765-020-00510-4.

Additional file 1: Figure S1. Emission spectra of LED light sources. Figure S2. Fresh weight of A. rugosa plantlets grown under LED lights. Results are given as the mean of three biological replicates \pm standard deviation.

\section{Abbreviations}

3'-H: Hydroxycinnamoyl; 3-H, hydroxycinnamoyl; 4CL: 4-Coumaryl-CoA ligase; A4OMT: Apigenin 4'-O-methyltransferase; $\mathrm{C4H}$ : Cinnamate 4-hydroxylase; CHI: Chalcone isomerase; CHS: Chalcone synthase; FS: Flavone synthase; GT: Glucosyltransferase; HPLC: High-performance liquid chromatography; HPPR: Hydroxyphenylpyruvate reductase; PAL: Phenylalanine ammonia-lyase; qRTPCR: Quantitative real-time PCR; RAS: Hydroxycinnamoyl-CoA; hydroxyphenyllactate hydroxycinnamoyl transferase; TAT: Tyrosine aminotransferase.

\section{Acknowledgements}

We thank Dr. Hyeong-Kyu Lee at the Korea Research Institute of Bioscience and Biotechnology (KRIBB), Daejeon, South Korea for providing tilianin standards.

\section{Authors' contributions}

SUP and JKK conceived and designed the experiments. WTP, SKY, RS and JSP performed the experiments and analyzed the data. WTP, RS, and SUP wrote the manuscript. All authors read and approved the final manuscript.

\section{Funding}

This research was supported by Basic Science Research Program through the National Research Foundation of Korea (NRF) funded by the Ministry of Education, Science, and Technology (20110010231) and by Korea Institute of Planning and Evaluation for Technology in Food, Agriculture, Forestry and Fisheries (IPET) through Advanced Production Technology Development Program (or Project), funded by Ministry of Agriculture, Food and Rural Affairs (MAFRA) (116115-03-1).

\section{Availability of data and materials}

Gene sequences were deposited to NCBI GenBank.

\section{Ethics approval and consent to participate}

Not applicable because we did not work with animals or humans.

\section{Competing interests}

The authors declare that they have no competing interests.

\section{Author details}

${ }^{1}$ Department of Crop Science, Chungnam National University, 99 Daehak-ro, Yuseong-gu, Daejeon 34134, South Korea. ${ }^{2}$ Department of Horticultural Science, Chungnam National University, 99 Daehak-ro, Yuseong-gu, Daejeon 34134, South Korea. ${ }^{3}$ Division of Life Sciences and Bio-Resource and Environmental Center, Incheon National University, Incheon 22012, South Korea.

\section{Received: 5 February 2020 Accepted: 5 May 2020}

Published online: 13 May 2020

\section{References}

1. Ahn B, Yang CB (1991) Volatile flavor components of Bangah (Agastache rugosa O. Kuntze) Herb. Kor J Food Sci Technol 23:582-586

2. Hong JJ, Choi JH, Oh SR, Lee HK, Park JH, Lee KY, Kim JJ, Jeong TS, Oh GT (2001) Inhibition of cytokine-induced vascular cell adhesion molecule-1 expression: possible mechanism for anti-atherogenic effect of Agastache rugosa. FEBS Lett 495:142-147. https://doi.org/10.1016/S0014 -5793(01)02379-1

3. Lee C, Kim H, Kho Y (2002) Agastinol and agastenol, novel lignans from Agastache rugosa and their evaluation in an apoptosis inhibition assay. J Nat Prod 65:414-416. https://doi.org/10.1021/np010425e

4. Kim HK, Lee HK, Shin CG, Huh H (1999) HIV integrase inhibitory activity of Agastache rugosa. Arch Pharm Res 22:520-523. https://doi.org/10.1007/ bf02979163

5. Shin S, Kang CA (2003) Antifungal activity of the essential oil of Agastache rugosa Kuntze and its synergism with ketoconazole. Lett Appl Microbiol 36:111-115. https://doi.org/10.1046/j.1472-765X.2003.01271.x

6. Choi KS, Lee HY (1999) Characteristics of useful components in the leaves of baechohyang (Agastache rugosa, O. Kuntze). J Kor Soc Food Sci Nutr 28:326-332

7. Lee HK, Oh SR, Kim Jl, Kim JW, Lee CO (1995) Agastaquinone, a new cytotoxic diterpenoid quinone from Agastache rugosa. J Nat Prod 58:1718-1721. https://doi.org/10.1021/np50125a011

8. Han DS (1987) Triterpenes from the root of Agastache rugosa. Kor J Pharmacogn 18:50-53

9. Oh HM, Kang YJ, Lee YS, Park MK, Kim SH, Kim HJ, Seo HG, Lee JH, Chang KC (2006) Protein kinase G-dependent heme oxygenase-1 induction by Agastache rugosa leaf extract protects RAW264.7 cells from hydrogen peroxide-induced injury. J Ethnopharmacol 103:229-235. https://doi. org/10.1016/j.jep.2005.08.030

10. Hernandez-Abreu O, Castillo-Espana P, Leon-Rivera I, Ibarra-Barajas M, Villalobos-Molina R, Gonzalez-Christen J, Vergara-Galicia J, Estrada-Soto S (2009) Antihypertensive and vasorelaxant effects of tilianin isolated from Agastache mexicana are mediated by NO/cGMP pathway and potassium channel opening. Biochem Pharmacol 78:54-61. https://doi. org/10.1016/j.bcp.2009.03.016

11. Nam KH, Choi JH, Seo YJ, Lee YM, Won YS, Lee MR, Lee MN, Park JG, Kim YM, Kim HC, Lee CH, Lee HK, Oh SR, Oh GT (2006) Inhibitory effects of tilianin on the expression of inducible nitric oxide synthase in low density lipoprotein receptor deficiency mice. Exp Mol Med 38:445-452. https:// doi.org/10.1038/emm.2006.52

12. Petersen M, Simmonds MSJ (2003) Molecules of interest-rosmarinic acid. Phytochemistry 62:121-125. https://doi.org/10.1016/S0031 -9422(02)00513-7

13. Dhabi NA, Arasu MV, Park CH, Park SU (2014) Recent studies on rosmarinic acid and its biological and pharmacological activities. Excli J 13:11921195. https://doi.org/10.17877/DE290R-6923

14. Gao LP, Wei HL, Zhao HS, Xiao SY, Zheng RL (2005) Antiapoptotic and antioxidant effects of rosmarinic acid in astrocytes. Pharmazie 60:62-65

15. Swarup V, Ghosh J, Ghosh S, Saxena A, Basu A (2007) Antiviral and antiinflammatory effects of rosmarinic acid in an experimental murine model of Japanese encephalitis. Antimicrob Agents Ch 51:3367-3370. https:// doi.org/10.1128/Aac.00041-07

16. Vogt T (2010) Phenylpropanoid biosynthesis. Mol Plant 3:2-20. https:// doi.org/10.1093/mp/ssp106

17. Jiang CG, Schommer CK, Kim SY, Suh DY (2006) Cloning and characterization of chalcone synthase from the moss, Physcomitrella patens. Phytochemistry 67:2531-2540. https://doi.org/10.1016/j.phyto chem.2006.09.030

18. Li F, Jin Z, Qu W, Zhao D, Ma F (2006) Cloning of a cDNA encoding the Saussurea medusa chalcone isomerase and its expression in transgenic tobacco. Plant Physiol Biochem 44:455-461. https://doi.org/10.1016/j. plaphy.2006.08.006

19. Martens S, Mithofer A (2005) Flavones and flavone synthases. Phytochemistry 66:2399-2407. https://doi.org/10.1016/j.phytochem.2005.07.013

20. Kuroki G, Poulton JE (1981) The para-O-methylation of apigenin to acacetin by cell-free extracts of Robinia pseudoacacia L. Z Naturforsch 36:916-920. https://doi.org/10.1515/znc-1981-11-1202

21. Ogata J, Itoh Y, Ishida M, Yoshida H, Ozeki Y (2004) Cloning and heterologous expression of cDNAs encoding flavonoid glucosyltransferases from Dianthus caryophyllus. Plant Biotechnol 21:367-375. https://doi. org/10.5511/plantbiotechnology.21.367

22. Petersen MS (1991) Characterization of rosmarinic acid synthase from cell cultures of Coleus blumei. Phytochemistry 30:2877-2881. https://doi. org/10.1016/S0031-9422(00)98217-7 
23. Petersen M (1997) Cytochrome P450-dependent hydroxylation in the biosynthesis of rosmarinic acid in Coleus. Phytochemistry 45:1165-1172 https://doi.org/10.1016/S0031-9422(97)00135-0

24. Ncube B, Finnie JF, Van Staden J (2012) Quality from the field: the impact of environmental factors as quality determinants in medicinal plants. $S$ Afr J Bot 82:11-20. https://doi.org/10.1016/j.sajb.2012.05.009

25. Cuong D, Ha TW, Park CH, Kim NS, Yeo HJ, Chun SW, Kim C, Park SU (2019) Effects of LED lights on expression of genes involved in phenylpropanoid biosynthesis and accumulation of phenylpropanoids in wheat sprout. Agronomy-Basel. https://doi.org/10.3390/agronomy9060307

26. Lian TT, Cha SY, Moe MM, Kim YJ, Bang KS (2019) Effects of different colored LEDs on the enhancement of biologically active ingredients in callus cultures of Gynura procumbens (Lour.) Merr. Molecules. https://doi. org/10.3390/molecules24234336

27. Lee SW, Seo JM, Lee MK, Chun JH, Antonisamy P, Arasu MV, Suzuki T, AlDhabi NA, Kim SJ (2014) Influence of different LED lamps on the production of phenolic compounds in common and Tartary buckwheat sprouts. Ind Crop Prod 54:320-326. https://doi.org/10.1016/j.indcrop.2014.01.024

28. Okamoto K, Yanagi T, Kondo S (1997) Growth and morphogenesis of lettuce seedlings raised under different combinations of red and blue light. Acta Hortic. https://doi.org/10.17660/actahortic.1997.435.14

29. Schuerger AC, Brown CS, Stryjewski EC (1997) Anatomical features of pepper plants (Capsicum annuum L) grown under red light-emitting diodes supplemented with blue or far-red light. Ann Bot-London 79:273-282. https://doi.org/10.1006/anbo.1996.0341

30. Seo JM, Arasu MV, Kim YB, Park SU, Kim SJ (2015) Phenylalanine and LED lights enhance phenolic compound production in tartary buckwheat sprouts. Food Chem 177:204-213. https://doi.org/10.1016/j.foodc hem.2014.12.094

31. Thwe AA, Kim YB, Li X, Seo JM, Kim SJ, Suzuki T, Chung SO, Park SU (2014) Effects of light-emitting diodes on expression of phenylpropanoid biosynthetic genes and accumulation of phenylpropanoids in Fagopyrum tataricum sprouts. J Agric Food Chem 62:4839-4845. https://doi. org/10.1021/jf501335q

32. Tuan PA, Thwe AA, Kim YB, Kim JK, Kim SJ, Lee S, Chung SO, Park SU (2013) Effects of white, blue, and red light-emitting diodes on carotenoid biosynthetic gene expression levels and carotenoid accumulation in sprouts of tartary buckwheat (Fagopyrum tataricum Gaertn.). J Agric Food Chem 61:12356-12361. https://doi.org/10.1021/jf4039937

33. Li X, Thwe AA, Park NI, Suzuki T, Kim SJ, Park SU (2012) Accumulation of phenylpropanoids and correlated gene expression during the development of tartary buckwheat sprouts. J Agric Food Chem 60:5629-5635. https://doi.org/10.1021/jf301449a

34. OuYang FQ, Mao JF, Wang JH, Zhang SG, Li Y (2015) Transcriptome analysis reveals that red and blue light regulate growth and phytohormone metabolism in Norway spruce [Picea abies (L.) Karst.]. PLoS ONE. https:// doi.org/10.1371/journal.pone.0127896

35. Johkan M, Shoji K, Goto F, Hashida S, Yoshihara T (2010) Blue light-emitting diode light irradiation of seedlings improves seedling quality and growth after transplanting in red leaf lettuce. HortScience 45:1809-1814. https://doi.org/10.21273/Hortsci.45.12.1809
36. Kim YJ, Kim YB, LiX Choi SR, Park S, Park JS, Lim YP, Park SU (2015) Accumulation of phenylpropanoids by white, blue, and red light irradiation and their organ-specific distribution in Chinese cabbage (Brassica rapa ssp Pekinensis). J Agric Food Chem 63:6772-6778. https://doi. org/10.1021/acs.jafc.5b02086

37. Ma G, Zhang LC, Kato M, Yamawaki K, Kiriiwa Y, Yahata M, Ikoma Y, Matsumoto H (2012) Effect of blue and red LED light irradiation on $\beta$-cryptoxanthin accumulation in the flavedo of citrus fruits. J Agric Food Chem 60:197-201. https://doi.org/10.1021/jf203364m

38. Zhang LC, Ma G, Kato M, Yamawaki K, Takagi T, Kiriiwa Y, Ikoma Y, Matsumoto H, Yoshioka T, Nesumi H (2012) Regulation of carotenoid accumulation and the expression of carotenoid metabolic genes in citrus juice sacs in vitro. J Exp Bot 63:871-886. https://doi.org/10.1093/jxb/err318

39. Rani A, Singh K, Sood P, Kumar S, Ahuja PS (2009) p-Coumarate:CoA ligase as a key gene in the yield of catechins in tea [Camellia sinensis (L.) $\mathrm{O}$. Kuntze]. Funct Integr Genomic 9:271-275. https://doi.org/10.1007/s1014 2-008-0098-3

40. Singh K, Kumar S, Rani A, Gulati A, Ahuja P (2009) Phenylalanine ammonia-lyase (PAL) and cinnamate 4-hydroxylase $(\mathrm{C} 4 \mathrm{H})$ and catechins (flavan-3-ols) accumulation in tea. Funct Integr Genomic 9:125-134. https ://doi.org/10.1007/s10142-008-0092-9

41. Xu F, Cai R, Cheng SY, Du HW, Wang Y, Cheng SH (2008) Molecular cloning, characterization and expression of phenylalanine ammonia-lyase gene from Ginkgo biloba. Afr J Biotechnol 7:721-729

42. Tuan PA, Park WT, Xu H, Park NI, Park SU (2012) Accumulation of tilianin and rosmarinic acid and expression of phenylpropanoid biosynthetic genes in Agastache rugosa. J Agric Food Chem 60:5945-5951. https://doi. org/10.1021/jf300833m

43. Hu YS, Zhang L, Di P, Chen WS (2009) Cloning and induction of phenylalanine ammonia-lyase gene from Salvia miltiorrhiza and its effect on hydrophilic phenolic acids levels. Chin J Nat Med 7:0449-0457

44. Song J, Wang ZZ (2011) RNAi-mediated suppression of the phenylalanine ammonia-lyase gene in Salvia miltiorrhiza causes abnormal phenotypes and a reduction in rosmarinic acid biosynthesis. J Plant Res 124:183-192. https://doi.org/10.1007/s10265-010-0350-5

45. Weitzel C, Petersen M (2010) Enzymes of phenylpropanoid metabolism in the important medicinal plant Melissa officinalis L. Planta 232:731-742. https://doi.org/10.1007/s00425-010-1206-x

46. Lin KH, Huang MY, Huang WD, Hsu MH, Yang ZW, Yang CM (2013) The effects of red, blue, and white light-emitting diodes on the growth, development, and edible quality of hydroponically grown lettuce (Lactuca sativa L. var. capitata). Sci Hortic-Amsterdam 150:86-91. https://doi. org/10.1016/j.scienta.2012.10.002

\section{Publisher's Note}

Springer Nature remains neutral with regard to jurisdictional claims in published maps and institutional affiliations.

\section{Submit your manuscript to a SpringerOpen ${ }^{\circ}$ journal and benefit from:}

- Convenient online submission

- Rigorous peer review

- Open access: articles freely available online

- High visibility within the field

Retaining the copyright to your article

Submit your next manuscript at springeropen.com 